[0212-7199(2002) 19: 2; pp 76-78]
ANALES DE MEDICINA INTERNA Copyright $\odot 2002$ ARAN EDICIONES, S.L.

An. Med InTERna (Madrid) AN. MED. INTERNA (Madrid)
Vol. 19, N. $^{2}$ 2, pp. $76-78,2002$

\section{Paraplejía por hipopotasemia secundaria a diarrea aguda}

\author{
F. ORTUÑO ANDÉRIZ, N. CABELLO CLOTET*, R. DE DIEGO GAMARRA, \\ I. SALAVERRÍA GARZÓN, S. VÁZQUEZ RIZALDOS \\ Servicios de Medicina Intensiva y *Medicina Interna IV. Hospital Clínico San Carlos de \\ Madrid
}

\author{
HIPOKALEMIC PARAPLEGIA DUE TO ACUTE DIARRHEA
}

\begin{abstract}
RESUMEN
La hipopotasemia puede dar una clínica variada pero es frecuente que curse de forma asintomática o con síntomas inespecíficos si es de escasa cantidad. En nuestro medio las causas de la hipopotasemia son variadas pero una de las etiologías más frecuentes en paises en vías de desarrollo son los cuadros diarreicos. Presentamos un cuadro de hipopotasemia severa debida a diarrea que se manifestó con intensa clínica neurológica pero que mejoró espectacularmente con tratamiento habitual.
\end{abstract}

PALABRAS CLAVE: Hipopotasemia. Paraplejia. Diarrea.

\begin{abstract}
Hipokalemia can give a variety of sintomatology but more often courses without it or with inespecifyc clinical manifestations. In our enviroment the etiology of hipokalemia is wide but one of the most com mon causes in third world countries are diarrheas. We describe a case of severe hipokalemia due to acute diarrhea which was maniffested with severe neurologyc sintoms but improves with conventional treatment.
\end{abstract}

KEY WORDS: Hipokalemia. Paraplegia. Diarrhea.

Ortuño Andériz F, Cabello Clotet N, de Diego Gamarra R, Salaverría Garzón I, Vázquez Rizaldos S. Paraplejia por hipopotasemia secunda ria a diarrea aguda. An Med Interna (Madrid) 2002; 19: 76-78.

\section{INTRODUCCIÓN}

Las parálisis periódicas son un grupo de enfermedades que pueden clasificarse en hiper, normo o hipocaliémicas. Existen formas primarias (como la parálisis periódica familiar hipocaliémica) y secundarias como las debidas al uso de diuréticos o cuadros diarreicos severos y las asociadas al hipertiroidismo.

Las diarreas secretoras son una causa frecuente de hipopotasemia. Sólo cuando son intensas y generalmente crónicas la depleción de potasio es lo suficientemente importante como para producir síntomas. Presentamos un caso de hipopotasemia secundaria a depleción por diarrea en un paciente con intensa clínica neurológica.

\section{CASO APORTADO}

Se trata de un varón de 30 años, de nacionalidad alemana, que estando de vacaciones en la República Dominicana comienza con cefalea, malestar general y 2-3 deposiciones líquidas al día sin productos patológicos. No fiebre, vómitos ni otra sintomatología asociada. Había hecho profilaxis para la Malaria. Unos días después comienza con debilidad progresiva, especialmente en pier- nas. Durante el vuelo de regreso a Alemania estos síntomas aumentan imposibilitándole andar o ponerse de pie con mialgias e intensísima astenia. Por este motivo es trasladado a nuestro Hospital desde el Aeropuero de Madrid a los 4 días de haberse iniciado el cuadro.

Exploración al ingreso: bien coloreado, hidratado y perfundido. Tensión arterial de 120/80 mmHg; Temperatura de 36,2 grados centígrados; Auscultación cardiopulmonar, abdomen y extremidades normales; fuerza en miembros superiores conservada; paresia 4/5 de predominio proximal en miembros inferiores que imposibilitaba la bipedestación. Sensibilidad conservada; Reflejos miotáticos abolidos en piernas;

Complementarios: hemograma normal; Creatinina de 1,1; Urea de 37; Calcio de 8,9; Fósforo de 1,7; Magnesio de 1,4 (en mg/dL); Sodio de 138; Potasio de 1,4; Cloro de 92; Dióxido de carbono sanguíneo total de 30,5 (en meq/L); Creatínfosfoquinasa de 2787; GOT de136 y GPT de 80 (en U/L); Gasometría: pH: 7,46; HCO3: 29,8 meq/L; Orina: pH: 6,5; Na: 104; K: 33; Cl: 155 (en meq/L). Creatinina: $241 \mathrm{mg} / \mathrm{dL}$; no proteinuria ni glucosuria; sedimento normal. L.C.R. 2 hematíes y 1 leucocito por $\mathrm{mm}^{3}$; Proteinas: 0,84 gramos por litro; Glucosa: 78 mg/dL; Electrocardiograma: ritmo sinusal; Radiografía de Tórax, normal;

Evolución: durante las primeras 24 horas se trató con Poliglobina ante la posibilidad de Síndrome de Guillain-Barré, además de suero-

Trabajo aceptado: 14 de junio de 2000

Correspondencia: Francisco Ortuño Andériz. Unidad de Cuidados Intensivos. Hospital Clínico San Carlos de Madrid. C/ Martín Lagos, s/n. 28040 Madrid. 
terapia y $125 \mathrm{meq} / 24 \mathrm{~h}$ de Cloruro potásico con mejoría progresiva del estado general desapareciendo el cuadro neurólogico. En el momento del alta a los 6 días del ingreso el paciente podía andar y estaba asintomático. Presentaba un Potasio sérico de 3,9 meq/L; Magnesio de 1,5 mg/dL y Creatínfosfoquinasa de $2700 \mathrm{U} / \mathrm{L}$. La diarrea había cedido sin tratamiento específico a los dos días del ingreso.

Dado que el paciente deseaba trasladarse a su país y ante la discordancia entre la severidad de la hipopotasemia y el cuadro diarreico banal se recomendó que continuara allí el estudio para descartar otras causas de hipopotasemia (Síndrome de Bartter del adulto, Síndrome de Gittelman, Nefropatías intersticiales, etc). Fue dado de alta con suplementos de Potasio y de Magnesio como tratamiento.

\section{DISCUSIÓN}

Casi todo el potasio que se ingiere diariamente se elimina por el riñón, excepto unos 10 milimoles que se pierden por sudor y heces, a no ser que haya diarrea. La absorción del mismo a nivel intestinal aumenta a un nivel casi igual al aumento del Potasio en la dieta (1). La Aldosterona no sólo aumenta la excreción urinaria de Potasio sino también la intestinal aunque en mucha menor cuantía (de 0,3 a 8,7 meq/24 h); el mecanismo que explique este intercambio entre Sodio y Potasio a nivel intestinal es desconocido (1).

Las diarreas son causa frecuente de hipopotasemia. El líquido diarreico contiene una concentración elevada de Potasio (30-60 milimoles por litro). Las diarreas que más hipopotasemia producen son las secretoras sobre todo las secundarias a tumores de pancreas secretores de péptido intestinal vasoactivo y las debidas al uso de laxantes, así como las secundarias a adenomas vellosos de colon $(1,2)$. La hipopotasemia ocurre en el $83 \%$ de los pacientes con diarreas secundarias a Vipomas, en el $21 \%$ de los pacientes con Síndrome Carcinoide y en el $10 \%$ de los enfermos con Carcinoma Medular de Tiroides (1). Se han descrito también en relación con diarreas infecciosas sobre todo por Yersinina Enterocolítica, Salmonella, Strongyloides y Giardia Lamblia $(3,4)$. El proceso de absorción del Potasio por parte del intestino no se suele alterar en las diarreas, pero las pérdidas digestivas del mismo aumentan por el mayor gradiente entre la luz intestinal y la pared debido a la secreción de ácido clorhídrico, a la existencia de aniones procedentes del metabolismo bacteriano de carbohidratos no reabsorbidos que lo arrastran y probablemente por Hiperaldosteronismo secundario (1). Asímismo la concentración de Potasio en las heces aumenta proporcionalmente al grado en que lo hace la cantidad de agua de la diarrea. Lógicamente la baja ingesta de Potasio en los estados diarreicos contribuye a la hipopotasemia.

En general, cuando la pérdida de Potasio es digestiva, el Potasio urinario es menor de 20-25 milimoles al dia. La depleción de volumen por la diarrea ocasiona un hiperaldosteronismo secundario que motiva una conservación de Sodio a expensas de perder Potasio lo que puede colaborar en la génesis de la hipopotasemia.

Además, al tratar el riñón de compensar la alcalosis metabólica mediante la eliminación de bicarbonato, éste se va a comportar en el túbulo renal como un anión no reabsorbible y arrastrará Potasio a pesar de estar el organismo deplecionado del mismo. En nuestro caso el Potasio urinario era de 33 miliequivalentes por litro hecho probablemente motivado por cierto componente de hiperaldosteronismo secundario a la pérdida de volumen intravascular; sin embargo el resto de datos analíticos no parecen apoyar esta circunstancia como son la ausencia de Hipertensión arterial y signos de baja volemia (lo que concuerda con diarreas de escasa cuantía).

Entre las causas digestivas de hipopotasemia, hay que contemplar también los vómitos persistentes y la aspiración gástrica, circustancias no presentes en el presente caso. La estimulación de la bomba de Sodio-Potasio ATP asa por hormonas tiroideas (Parálisis periódicas tireotóxicas), Insulina o Adrenalina pueden colaborar en el desarrollo de la Hipopotasemia al introducir Potasio dentro de las células musculares, hígado y riñón (2,5-7).

La diarrea persistente puede conducir también a hipomagnesemia. Tanto el jugo gástrico como el intestinal son muy ricos en este ión. Es muy raro encontrarla aisladamente ya que se suele asociar a hipocalcemia e hipopotasemia. En concreto es muy frecuente la asociación entre hipomagnesemia e hipopotasemia (la hipomagnesemia está presente en el $40 \%$ de los pacientes con hipokaliemia) en parte porque las causas que conducen a ambas son las mismas, y en parte porque la hipomagnesemia facilita la excreción renal de Potasio probablemente por deplección de Adenosín Trifosfato (ATP) y apertura de los canales para el Potasio en las células tubulares (4). En estos casos la hipocaliemia suele ser refractaria al tratamiento con Potasio si préviamente no se ha administrado Magnesio. En este caso el tratamiento con suplementos de Potasio y Magnesio se instauró simultáneamente.

El estudio de las alcalosis metabólicas se puede simplificar con la determinación del Cloro urinario. Si éste es menor de 10 miliequivalentes por litro se habla de una alcalosis salino sensibles (por pérdida de jugo gástrico, adenomas vellosos o consumo reciente de diuréticos), pero si es mayor de 20 miliequivalentes por litro (como en el caso que nos ocupa) serán alcalosis metabólicas salino resistentes, en cuyo caso una determinación del Potasio en orina nos será de mucha utilidad ya que si éste es mayor de 30 miliequivalentes por litro y el paciente presenta cifras tensionales elevadas la sospecha será de hiperaldosteronismo. En nuestro caso de un paciente normotenso y Potasio en orina en torno a 30 miliequivalentes por litro en orina lo más probable es que se trate de una alcalosis metabólica secundaria a la hipopotasemia y/o hipomagnesemia. Otras posibilidades a considerar serían el abuso de laxantes o el Síndrome de Bartter.

La clínica de la hipopotasemia puede ser inexistente si es de instauración lenta y de pequeña intensidad. Las manifestaciones más frecuentes son las neuromusculares. Con concentraciones séricas de 2 a 2,5 milimoles por litro de Potasio existe debilidad muscular sobre todo en miembros inferiores. Si la hipopotasemia es importante se presenta paresia con arreflexia que puede conducir a parálisis respiratoria y la necesidad de uso de un respirador (8).

Es conocido que la hipopotasemia puede producir un cuadro de rabdomiolisis $(5,8-10)$. Entre las serie publicadas españolas son muy pocos los casos atribuidos a la hipocaliemia predominando las rabdomiolisis traumáticas, las debidas a alcoholismo, las miopatías inflamatorias, tras ejercicio intenso, etc. (9). En cualquier forma de rabdomiolisis se ha visto una excelente correlación entre la cifra de Creatínfosfokinasa y de Mioglobina plasmática, lo que es de suma utilidad para monitorizar la evolución de la enfermedad $(9,10)$. 
La hipocaliemia puede causar la llamada miopatía hipocaliémica de mecanismo no bien aclarado y cuya clínica puede variar desde debilidad, hinchazón, dolor y aumento de sensibilidad hasta desarrollo de tetraparesia aguda con rabdomiolisis secundaria $(4,5,7,8,11,12)$. Se postula que la depleción de Potasio puede causar vasoconstricción en varios lechos vasculares lo que impediría la respuesta fisiológica de hiperemia en el ejercicio, apoyando una etiología isquémica en la rabdomiolisis hipopotasémica (8). Sin embargo se han descrito rabdomiolisis francas en pacientes hipocaliémicos en ausencia de ejercicio (8). Probablemente la mayoría de los pacientes con rabdomiolisis de cualquier etiología no presentan necrosis celular histológica própiamente dicha sino que el trastorno radica en un daño funcional de la membrana del miocito lo que condiciona la salida de enzimas musculares al exterior (10) ocasionando elevación de Creatínfosfokinasa (CPK), Aspartatoaminotransferasa (AST), Alaninoaminotransferasa (ALT), y Lactodeshidrogenasa (LDH). Existirá también Mioglobinemia, mioglobinuria y gránulos pigmentados en el sedimento urinario. Así mismo puede afectarse el músculo liso dando ileo paralítico. Las alteraciones electrocardiográficas son frecuentes pero sin relación clara con los niveles de Potasio aunque se han descrito algunos casos de parada cardiaca asociados a estados hipòcaliémicos severos (7).

A nivel renal, la hipopotasemia altera la función tubular con disminución de la capacidad de concentración lo que conduce a poliuria. En las formas crónicas se originan cambios estructurales en las células tubulares con vacuolización de las mismas. El filtrado glomerular es normal o está ligeramente disminuido y en el análisis de orina se observan alteraciones mínimas en el sedimento.

El tratamiento de la hipopotasemia se basa en aportar Potasio a una velocidad apropiada a la gravedad clínica. En personas asintomáticas basta la vía oral estando el Acetato Potásico contraindicado en caso de coexistir la hipopotasemia con alcalosis metabólica pero en graves parálisis musculares la vía de administración deberá ser intravenosa y a una velocidad que no supere los 20 miliequivalentes por hora por una via central y siempre con estricta monitorización cardiaca. En nuestro caso se administraron más de 700 miliequivalentes de Cloruro Potásico durante el ingreso.

Para evitar el Fracaso Renal Agudo debemos infundir de 3 a 5 litros de Suero al día y conseguir una diuresis alcalina. En ocasiones son necesarias algunas sesiones de diálisis pero casi siempre con recuperación completa de la función renal.

\section{Bibliografía}

1. Agarwal R, Afzalpurkar R, Fordtran J. Pathophysiology of Potassium Absorption and Secretion by the Human Intestine. Gastroenterology, 1994; 107 (2): 548-751.

2. Fernández-Sola J, Pedrol E, Masanes F, Galofre J, Casademont J, Grau JM, Urbano-Márquez A. Parálisis periódica tireotóxica normocaliémica. Ann Med Interna (Madrid) 1992; 9: 294-296.

3. Cervelló A, Alfaro A, Chumillas MJ. Hypokalemic Myopathy Induced by Giardia Lamblia. N Engl J Med 1993; 329 (3): 210-211.

4. Orman RA, Lewis JB. Flaccid Quadriparesis Associated with Yersinia Enterocolitis-Induced Hypokalemia. Arch Intern Med 1989; 149: 1193-1194.

5. García I, Ciudad J, Moreno I, Martín T, del Cañizo F, de Diego JM Parálisis periódica tirotóxica en un varón español. Rev Clin Esp 1995; 195: 302-303.

6. Karantzias J, Giovannoni A, Lago C, Diab M, Bagilet D. Paraparesia hipopotasémica por hipertiroidismo iatrógeno. Rev Clin Esp. 1995; 195 : 360-361.
7. hipocaliémica tirotóxica: 18 casos por diferentes formas de tirotoxicosis. Rev Clin Esp. 1995; 195: 294-297.

8. Berlango A, Jiménez L, Montero FJ, Muñoz JA, Torres J, Calderón de la Barca JM. Rabdomiolisis aguda y tetraparesia secundarias a hipocaliemia por ingesta de regaliz. Ann Med Interna (Madrid) 1995; 12: 33-35.

9. Fernández-Solá J, Grau J.M, Pedro-Botet JC, Casademont J, Estruch R, Company X, Urbano-Márquez A. Rabdomiolisis no traumática: análisis clínico y morfológico de 53 casos. Med Clin (Bar) 1988; 90; 199-202.

10. Pascual JM, Górriz JL, Prosper M, Collado A, Belda A, Michavila J, Redón J. Rabdomiolisis Aguda. Estudio de 44 casos. Rev Clin Esp 1990; 187: 6-9.

11. Miró O, Pedrol E, Nogué S, Cardellach F. Hiponatremia e hipopotasemia graves inducidas por el consumo de Equisetum Telmateia. Med Clin (Barc.) 1996; 106 (16): 639

12. Neuman G, Kopman F. Dyskalemic Periodic Paralysis and Myotonia. Anesth Analg 1993; 76(2): 426-428. 